\title{
Catastro 3D: sistemas de referencia altimétrica para parcelas y objetos territoriales
}

\author{
Diego Alfonso Erba* \\ Gustavo Noguera** \\ Aldo Mangiaterra**
}

Recibido el 28 de abril de 2015; aceptado el 16 de agosto de 2015

\begin{abstract}
This paper describes the distinct existing systems to reference the height of objects and its possible applications in the structuring of 3D cadastre. It discusses advantages and inconveniences in the utilization of each one of them for the positioning of terrestrial objects in space.

Hypotheses based on similar studies of cases in 3D cadastre are analyzed, without the pretension of covering the totality in the same manner. The developed theory is applied to position apartments that are part of private residential buildings (3D parcels) and elements of road work (3D land objects), demonstrating the correlations between the distinct systems of heights.

It is possible to conclude from the results that the ellipsoid of revolution is the most appropriate reference surface for 3D parcels and generally all 3D land object heights. Furthermore, for certain territorial 3D objects, it may be necessary to utilize an equipotential surface to reference their heights.

Regarding the precisions required for the positioning and sizing of the plots and $3 \mathrm{D}$ objects, it is concluded that both must be carefully analyzed and defined in the cadastral legislation. It is not possible to set a single precision for all, parcels and land objects, since the accuracies depend of the function of the cadastral object.

Key words: $3 D$ cadastre, high for $3 D$ parcel, high for $3 D$ territorial objects.

* Centro de Vinculación de Estudios Territoriales, Universidad Nacional de Córdoba, Sede Ciudad Universitaria, Av. Vélez Sarsfield 1611, Córdoba, Argentina, correo electrónico: diegoerbalac@ gmail.com

** Universidad Nacional de Rosario, Av. Pellegrini 250, Rosario, Argentina, correo electrónico: agrim.noguera@gmail.com

*** Grupo de Geodesia Satelital de Rosario, Vélez Sarsfield 281, Rosario, Argentina, correo electrónico: aldomangiaterra@gmail.com
\end{abstract}




\section{Resumo}

O presente trabalho descreve os diferentes sistemas existentes para referenciar alturas de parcelas e objetos territoriais, bem como as possíveis aplicações na estruturação de cadastros 3D. Discute vantagens e inconvenientes derivados do uso de cada um para o posicionamento no espaço.

Hipóteses baseadas em estudos de casos similares de cadastro 3D são analisadas, sem ter a pretensão de cobrir a totalidade dos mesmos. A teoria desenvolvida é aplicada para posicionar apartamentos que fazem parte de prédios de moradias privadas (parcelas 3D) e elementos de uma ponte (objeto territorial 3D), mostrando as correlações entre os diferentes sistemas de alturas.

A partir dos resultados é possível concluir que o elipsoide de revolução é a superfície mais apropriada para referenciar as alturas das parcelas $3 \mathrm{D}$ e a maioria dos objetos territoriais $3 \mathrm{D}$, sendo que para determinados objetos pode ser necessário utilizar, além do elipsoide, uma superfície equipotencial para referenciar as alturas.

Em relação às precisões requeridas para o posicionamento e o dimensionamento das parcelas e objetos territoriais 3D, se conclui que ambas devem ser analisadas cuidadosamente e definidas na legislação cadastral. Não é possível fixar uma única precisão para todos, dado que estas devem ajustar-se em função de cada objeto cadastral.

Palavras chave: cadastro $3 D$, altura para parcela $3 D$, altura para objeto territorial $3 D$.

\section{Resumen}

El presente trabajo describe los distintos sistemas existentes para referenciar las alturas de parcelas y objetos territoriales, y sus posibles aplicaciones en la estructuración de catastros 3D. Discute ventajas e inconvenientes en la utilización de cada uno de ellos para el posicionamiento en el espacio.

A lo largo del desarrollo se analizan las hipótesis en estudios de casos propios del catastro 3D, sin la pretensión de cubrir la totalidad de los mismos. La teoría desarrollada es aplicada para posicionar departamentos que forman parte de edificios de viviendas privadas (parcelas 3D) y elementos de una obra vial (objetos territoriales 3D), mostrando las correlaciones entre los distintos sistemas de alturas.

A partir de los resultados es posible concluir que el elipsoide de revolución es la superficie más apropiada para referenciar las alturas de parcelas 3D y de la mayoría de los objetos territoriales $3 \mathrm{D}$, siendo que para determinados objetos territoriales $3 \mathrm{D}$ puede ser necesario utilizar, además del elipsoide, una superficie equipotencial para referenciar sus alturas.

En relación a las precisiones requeridas para el posicionamiento y dimensionamiento de las parcelas $3 \mathrm{D}$ y de los objetos $3 \mathrm{D}$, se concluye que ambas deben ser 
analizadas cuidadosamente y definidas en la legislación catastral. No es posible fijar una única precisión para todos, puesto que estas deberán ajustarse a la función de cada objeto catastral.

Palabras clave: catastro $3 D$, alturas para parcela $3 D$, alturas para objeto territorial $3 D$.

\section{Introducción}

El Catastro 3D debe registrar los objetos territoriales con precisión en el espacio y en el tiempo, identificándolos y ubicándolos geométricamente como volúmenes en un determinado momento. Un registro 3D permite relacionar objetos territoriales, o parte de ellos, reposicionarlos retrospectivamente, proyectar modificaciones y analizar la influencia de nuevos objetos aún antes de que existan a través de la construcción de escenarios prospectivos.

El Catastro 3D, más allá de las medidas del objeto territorial, debe contar con las tres coordenadas de un número suficiente de puntos que permitan ubicarlo espacialmente como bloque en el marco de referencia y a la fecha de medición. El número de puntos de georreferenciación dependerá de cada caso y será definido por el profesional de acuerdo a su criterio y experiencia, de manera que se garanticen los parámetros de precisión establecidos por la norma catastral.

El posicionamiento planimétrico de los objetos territoriales no presenta mayor dificultad una vez que se establece el sistema de referencia geodésico, no obstante, la definición de la superficie de referencia más adecuada para determinar las alturas, está aún en discusión.

El presente trabajo describe distintos sistemas existentes para referenciar las alturas de parcelas y objetos territoriales, y sus posibles aplicaciones en la estructuración de catastros 3D. Discute ventajas e inconvenientes en la utilización de cada uno de ellos para el posicionamiento en el espacio.

A lo largo del desarrollo se analizan las hipótesis en estudios de casos propios del catastro 3D, sin la pretensión de cubrir la totalidad de los mismos. La teoría desarrollada es aplicada para posicionar departamentos que forman parte de edificios de viviendas privadas (parcelas 3D) y elementos de una obra vial (objetos territoriales 3D), mostrando las correlaciones entre los distintos sistemas de alturas.

Los resultados alcanzados en esta investigación permiten afirmar que el elipsoide de revolución es la superficie más apropiada para referenciar las alturas de parcelas $3 \mathrm{D}$ y de la mayoría de los objetos territoriales $3 \mathrm{D}$, siendo que para determinados objetos territoriales 3D puede ser necesario utilizar, además del elipsoide, una superficie equipotencial para referenciar sus alturas. También permiten afirmar la posibilidad de construir modelos "locales" de geoide que permitan convertir diferencias de alturas elipsoidales en diferencias de alturas geoidales (y viceversa). 
Lo aquí desarrollado cobra mayor importancia a partir de la aplicación del nuevo Código Civil y Comercial y la consecuente ampliación de derechos reales en Argentina.

\section{Sistemas de alturas para Catastro 3D}

Una superficie de referencia es aquella a partir de la cual se definen las alturas de puntos en el espacio. Para establecerla es necesario fijar diferentes parámetros, siendo los planos, los elipsoides de revolución y las superficies equipotenciales las más comúnmente usadas.

Un plano horizontal es la superficie de referencia más simple en términos geométricos y de más fácil comprensión para los usuarios. Su posición espacial se define como normal a la vertical en un punto origen y las alturas de los demás puntos se miden a lo largo de rectas perpendiculares a ese plano. Desde que, en términos generales, se considera vertical de un punto a la dirección de la fuerza de gravedad terrestre que pasa por él, las rectas perpendiculares al plano de referencia que se adopte y las verticales no necesariamente coinciden. Por otra parte las alturas resultan válidas sólo para extensiones reducidas puesto que a $1 \mathrm{~km}$ de distancia del punto origen el error altimétrico ya es del orden de $10 \mathrm{~s} 8 \mathrm{~cm}$ debido a la curvatura terrestre.

Un elipsoide de revolución es una superfície de referencia de carácter matemático; su centro, orientación, semieje mayor y aplastamiento son elegidos de tal forma que su aproximación al geoide sea máxima.

Una superficie equipotencial o superficie de nivel es siempre perpendicular a la vertical, y dado que la dirección de las verticales es influenciada por la distribución de masas y por el movimiento terrestre, esta superficie resulta ser irregular. Se adopta como superficie equipotencial de referencia al geoide que es la que mejor se adapta al nivel medio del mar a escala global.

Las distancias de los puntos en el espacio hasta las superficies de referencia se denominan alturas, aun cuando en el lenguaje geodésico frecuentemente son denominadas cotas.

Dado que las superficies equipotenciales no son paralelas, el resultado de una nivelación geométrica dependerá del camino recorrido.

La Figura 1 muestra que la distancia a lo largo de la vertical entre las superficies equipotenciales $W_{A}$ y $W_{B}$ no es la misma si se mide en A que si mide en $\mathrm{B}$ o si se recorre el camino por medio de sucesivas estaciones de nivel siguiendo determinado itinerario.

Al variar el itinerario puede variar el resultado. La ambigüedad de las cotas obtenidas de tal forma conduce a la denominación de alturas brutas. 


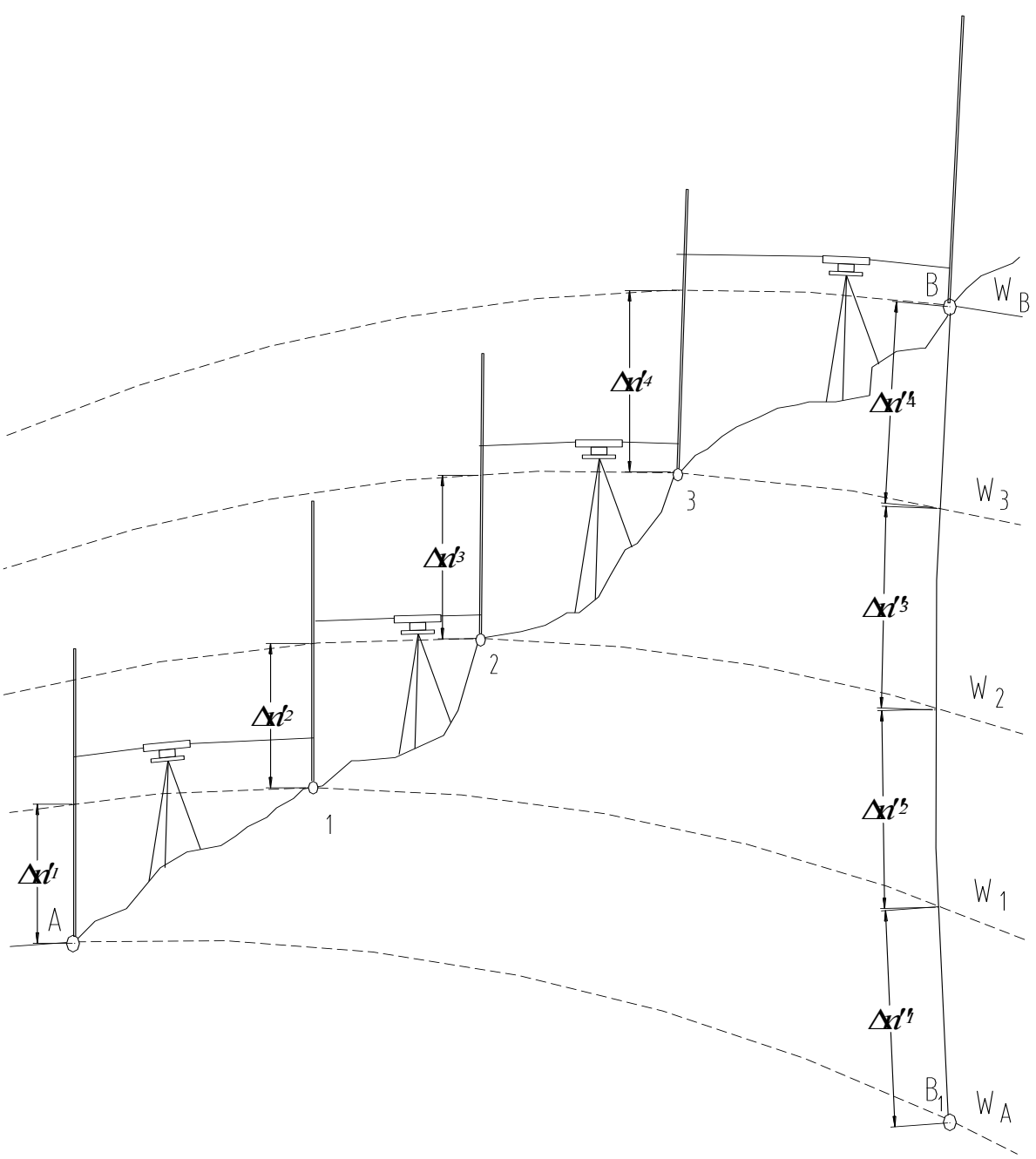

Figura 1. Distancias entre superficies equipotenciales.

El concepto de nivel se puede asociar también al concepto de potencial gravitatorio, puesto que puntos ubicados a mayor altura tienen un potencial gravitatorio menor. Considerando como superficie equipotencial de referencia al geoide de potencial $W_{0}$ (origen del mareógrafo), se define como cota geopotencial del punto $\mathrm{P}$ a la diferencia entre el potencial en $W_{0}$ y el potencial de la superficie de nivel que pasa por $P\left(W_{P}\right)$ (Figura 2$)$. 

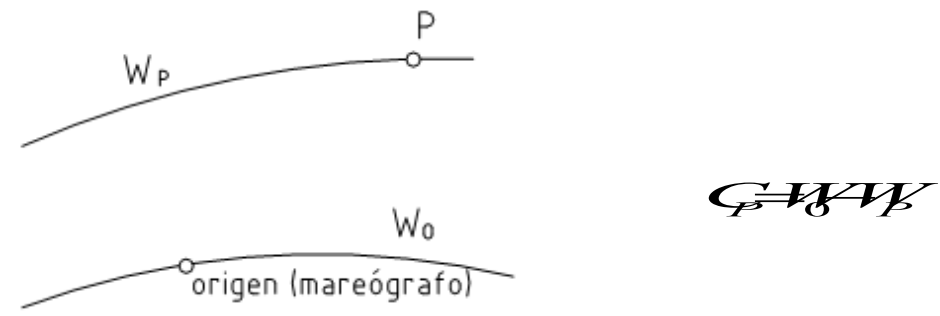

Figura 2. Cota geopotencial.

Las cotas geopotenciales determinan rigurosamente la dirección de escurrimiento del agua entre los puntos y el valor que se obtiene para cualquier punto es independiente del camino utilizado, razón por la cual en todos los casos se obtendrá un resultado único. Cabe señalar que las cotas geopotenciales no representan una longitud sino el trabajo necesario para trasladar una masa unitaria desde el origen 0 hasta un punto $P$. Esta última característica hace que, en la práctica, su utilización no sea conveniente en levantamientos terrestres.

Para evitar esta limitación, Huerta (2001) nos recuerda la definición de las cotas dinámicas $(C D)$ como el cociente entre la cota geopotencial y un valor de gravedad constante. De esta manera los valores resultantes se expresan como distancias. Normalmente se utiliza el valor de gravedad normal sobre el elipsoide a altitud $0 \mathrm{~m}$ y latitud de $45^{\circ}$.

$$
C D=C_{P}^{d i n}=\frac{C_{P}}{\gamma_{0}^{45}}
$$

Así, las cotas dinámicas tienen una característica común con las cotas geopotenciales: los puntos de igual cota pertenecen a la misma superficie equipotencial. Esta característica hace que, aunque se expresan en unidades de distancia, resulten insensibles a la convergencia de las superficies de nivel. Para determinar la cota geopotencial o la dinámica se debe realizar una nivelación geométrica midiendo a la vez la aceleración de la gravedad $(g)$.

Entendiendo claramente los conceptos de cotas, pasamos a los conceptos de alturas, las cuales son utilizadas para el posicionamiento de las parcelas y los objetos 3D (Figura 3).

La altura ortométrica $(H)$ de un punto ubicado en la superficie terrestre es la distancia entre él y la superficie equipotencial de referencia (geoide), medida a lo 
largo de la dirección de la vertical. Esta altura se obtiene a partir de la cota geopotencial considerando un valor medio de g, lo que implicaría conocer la distribución de masas entre el punto en cuestión y su proyección (según la vertical) sobre el geoide. Esto en rigor resulta imposible, pero se puede suplir satisfactoriamente por un cálculo utilizando parámetros conocidos.

La altura normal es determinada a partir de la gravedad normal en vez de un valor medio de g, es decir, surge de un cálculo que considera un modelo adoptado para el campo gravitatorio terrestre. La altura normal es un valor que poco difiere de la altura ortométrica (en general pocos centímetros), y define una nueva superficie de referencia denominada cuasi-geoide. Se puede afirmar que la separación entre el geoide y el cuasi-geoide varía (tomando casos extremos) de algunos centímetros a poco más de un metro. Para la superficie de los océanos el geoide y el cuasi-geoide son coincidentes.

La altura elipsoidal $(h)$ es la distancia entre un punto en el espacio y la superficie del elipsoide, medida a lo largo de la normal al elipsoide. El Sistema Global de Navegación Satelital (por su sigla en inglés Global Navigation Satellite Systems - GNSS) permite conocer esta altura con la precisión que fuere necesaria, inclusive de hasta pocos milímetros.

Al vincular $h$ y $H$ de un mismo punto es posible relacionar el geoide con el elipsoide a través de la ondulación del geoide $(N)$, donde: $\mathrm{N}=\mathrm{h}-\mathrm{H}$. En rigor, aun cuando la vertical del punto y la normal al elipsoide no son exactamente coincidentes, son tan próximas que su desvío no influye en el cálculo de N. Navratil y Unger (2011) afirman que para utilizar nivelación geométrica en la estimación de

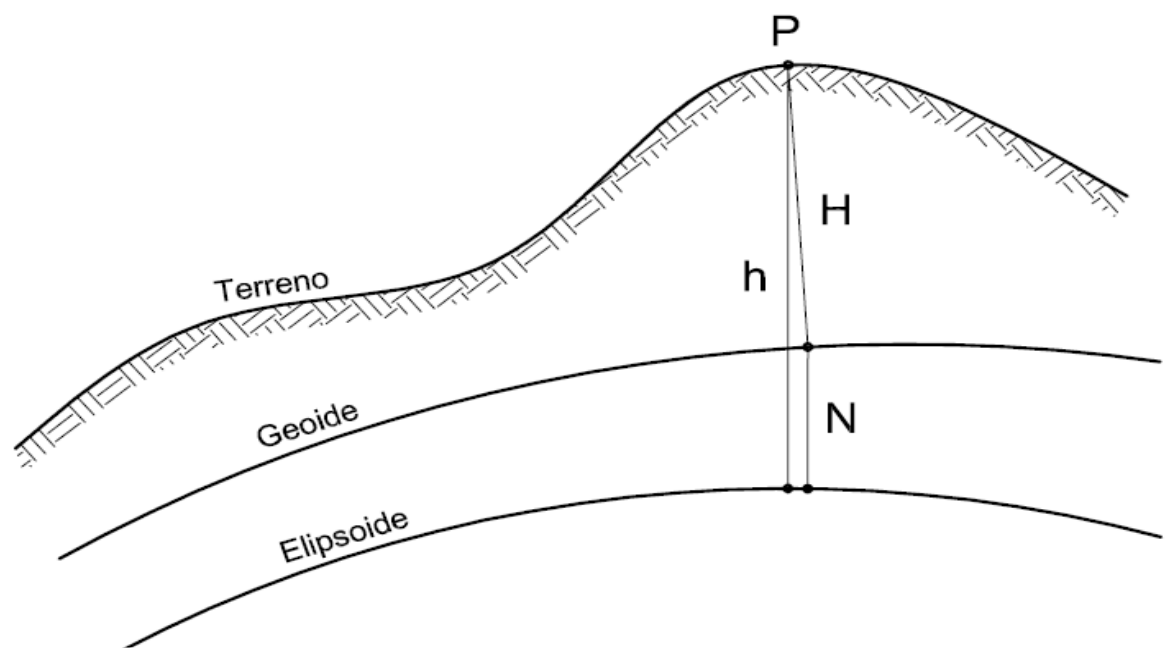

Figura 3. Alturas. 
diferencias de alturas elipsoidales es necesario conocer el desvío de la vertical. Esta afirmación es verdadera pero tiene un carácter muy general pues en la práctica depende de la precisión requerida y de la extensión que se quiere abarcar.

En zonas de llanura y en áreas cuyo radio no exceda algunas decenas de kilómetros es posible obtener modelos locales de geoide que permitan correlacionar las alturas ortométricas con las alturas elipsóidicas. Esta estrategia puede ser aplicada tanto en la construcción de un túnel, con posicionamiento satelital solamente en los extremos y nivelación geométrica en su interior, cuanto en la construcción de obra vial sobre superficie acuática que imposibilita la nivelación geométrica (salvo en los extremos). Apelando a una combinación inteligente de nivelación geométrica, posicionamiento satelital y modelización geoidal, los resultados pueden ser satisfactorios para el posicionamiento espacial de esos objetos territoriales.

En este caso, la expresión a utilizar para calcular desniveles es: $\left(\mathrm{H}_{2}-\mathrm{H}_{1}\right)=\left(\mathrm{h}_{2}-\mathrm{h}_{1}\right)$ - $\left(\mathrm{N}_{2}-\mathrm{N}_{1}\right)$.

Esa solución fue aplicada durante la construcción de la obra vial que une las ciudades argentinas de Rosario y Victoria, atravesando el delta del río Paraná de $60 \mathrm{~km}$ de ancho (Figura 4). Al comenzar la obra hubo una creciente extraordinaria del río que cubrió de agua toda el área, lo que hacía casi imposible realizar una nivelación geométrica.

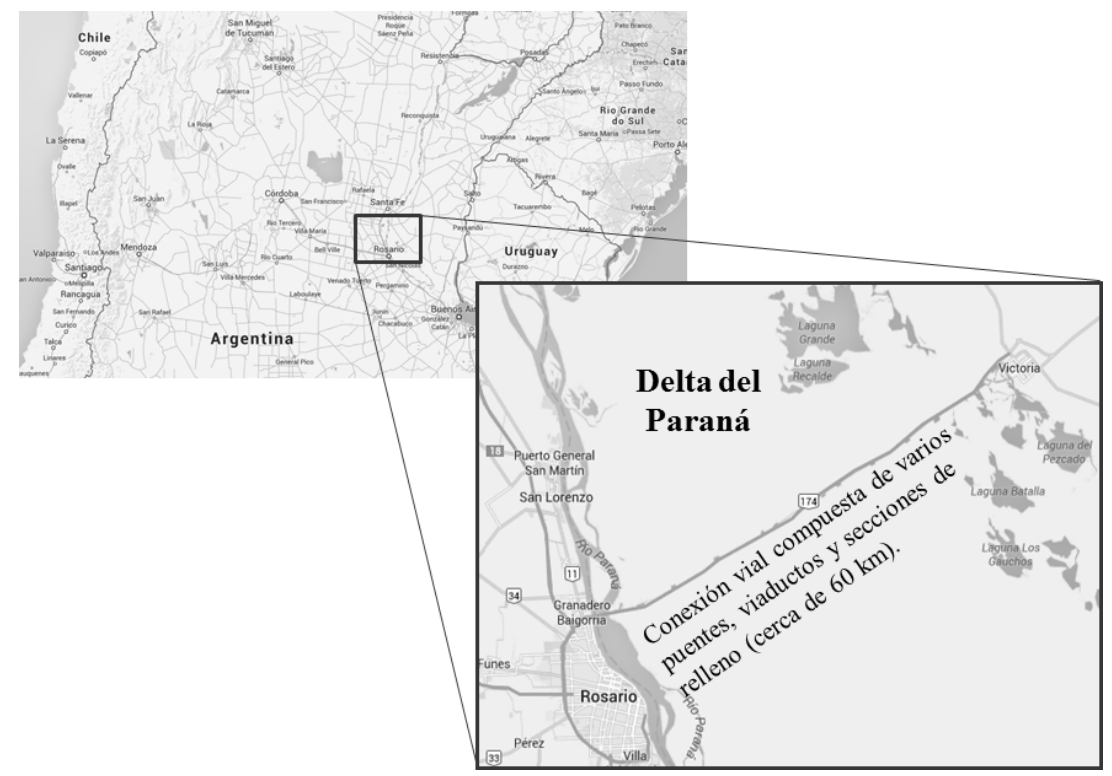

Figura 4. Ubicación del área de estudio, Rosario, Argentina. Fuente: adaptada de Google Maps. 
En ambas cabeceras se midieron las alturas elipsóidicas y sobre el nivel del mar y se determinaron los respectivos valores de N. Se midió también la gravedad en diversos puntos y, apelando a un modelo de geoide global, se pudo calcular la variación de $\mathrm{N}$ a partir del trazado de un perfil geoidal a lo largo de la traza de la obra. Siguiendo esta estrategia se pudieron obtener alturas sobre el nivel del mar en cualquier punto de la obra tomando como base el posicionamiento satelital, satisfaciendo una tolerancia de $10 \mathrm{~cm}$, lo cual se verificó una vez finalizada la construcción.

\section{Modelos de geoide y estaciones permanentes GNSS}

Para relacionar alturas elipsoidales y ortométricas se utilizan modelos de transformación de alturas denominados modelos de geoide, de los cuales es posible obtener $\mathrm{N}$ para la zona en estudio. Estos modelos pueden obtenerse a partir de la información de puntos geodésicos con ambas alturas ( $\mathrm{h} \mathrm{y} \mathrm{H}$ ), que se encuentran en la zona, generalmente de extensión limitada. Un ejemplo de este tipo es el Modelo de geoide de la ciudad de Rosario (MGR06) presentado en la Figura 5.

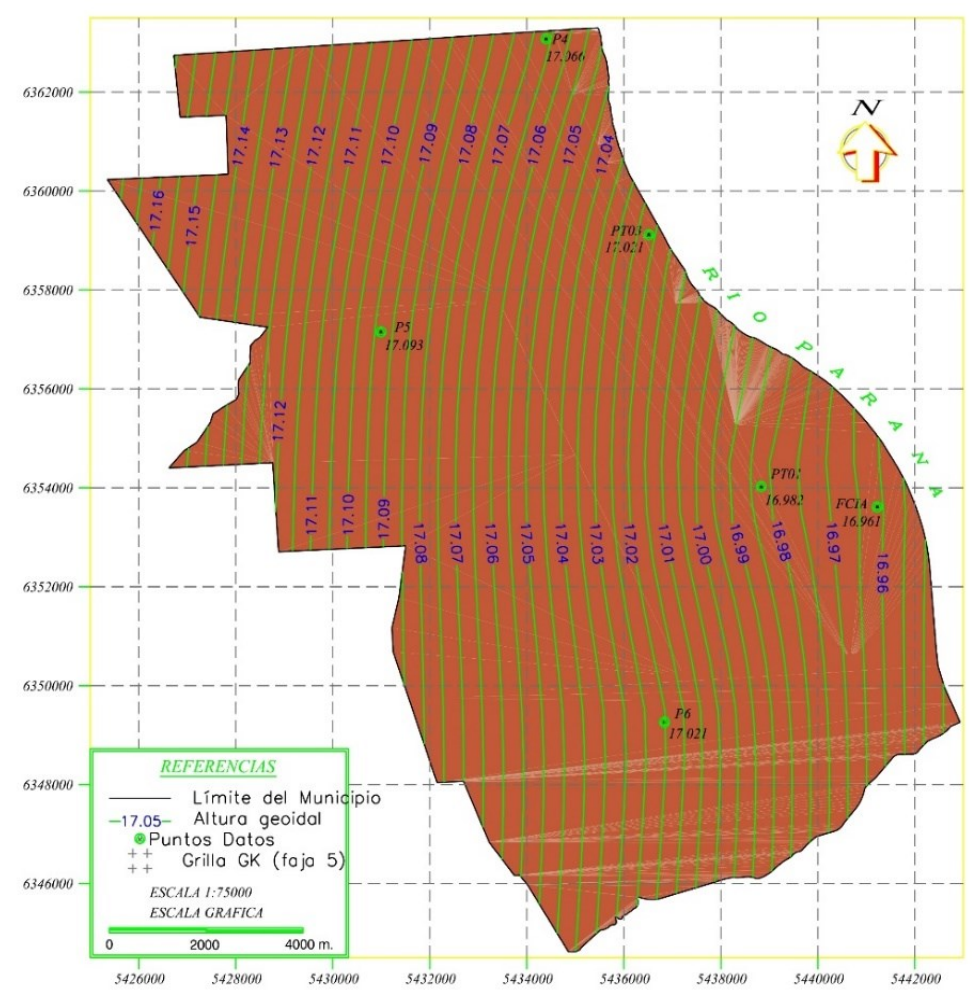

Figura 5. Modelo de geoide de la ciudad de Rosario, Argentina (MGR06).

Fuente: Balbarani et al. (2006). 
La relación de alturas elipsoidales y ortométricas puede determinarse también a partir de modelos globales como el EGM2008 (Earth Gravitational Model) y EIGEN (European Improved Gravity Model of the Earth by New Techniques), los cuales se construyen generalmente a partir de datos de observaciones de satélites específicos para este fin, y suelen tener como limitante la definición del "origen". Si se cuenta con uno (o varios) puntos con la doble información ( $\mathrm{h} \mathrm{y} \mathrm{H}$ ) en la zona, es posible calibrar el modelo global a partir del $\mathrm{N}$ de una estación permanente siendo el resultado útil para una zona amplia en estudio.

En la ciudad de Rosario y zonas aledañas, el EGM2008 fue calibrado con el N de la estación permanente denominada UNRO, el resultado de este trabajo está representado en la Figura 6.

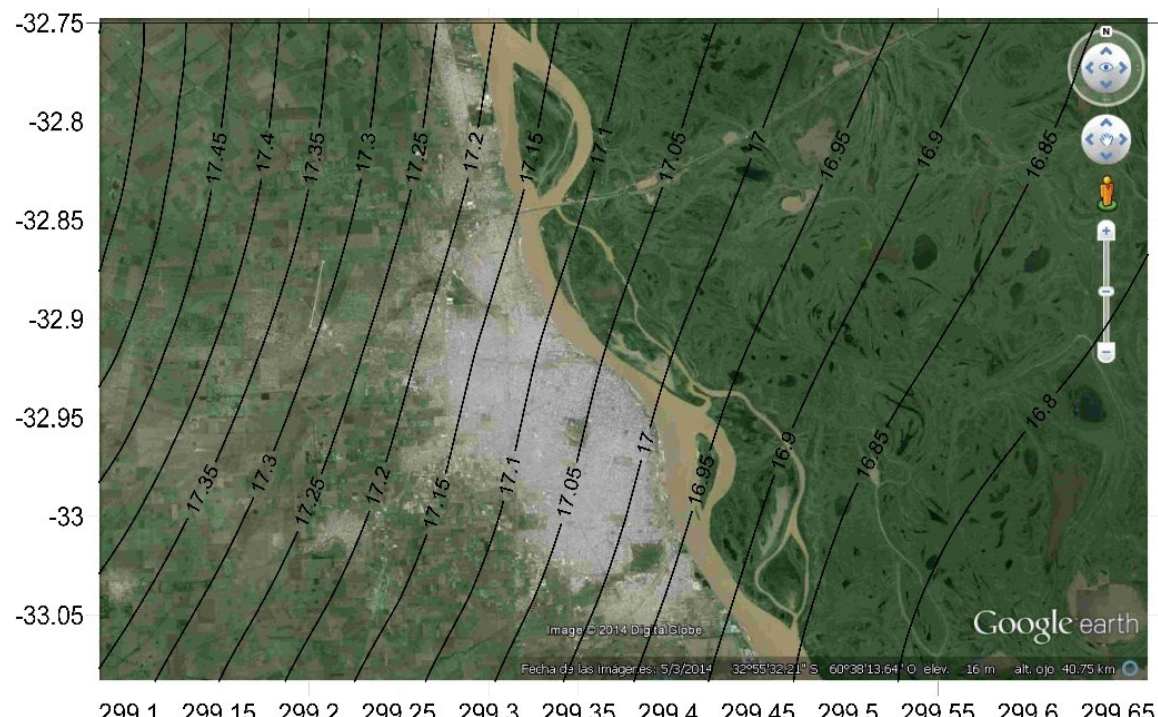

Figura 6. EGM2008 calibrado con el $\mathrm{N}$ de estación permanente UNRO.

Una estación permanente consiste en un receptor GNSS rastreando todos los satélites visibles, en forma continua las 24 horas, junto con un sistema de almacenamiento y publicación de los datos (generalmente a través de Internet, en tiempo real o en forma diferida) de forma que esos datos estén a disposición de los usuarios.

El conjunto de las estaciones permanentes conforman una red. En Argentina, la Red Argentina de Monitoreo Satelital Continuo (RAMSAC) está compuesta por algo más de 70 estaciones y es administrada por el Instituto Geográfico Nacional (IGN). Para que los datos de las estaciones permanentes resulten de utilidad a la georreferenciación, las coordenadas geodésicas (latitud, longitud y altura elipsóidi- 
ca h) de la estación deben ser conocidas y publicadas. Si todas las estaciones permanentes contaran con esta información (h y $\mathrm{H}$ ) se podría desarrollar de forma bastante simple un modelo geoidal de alcance regional o bien utilizar el valor de $\mathrm{N}$ en la estación para calibrar un modelo global para la zona de la estación. Contar con la coordenada $\mathrm{H}$ en el mismo punto de la estación sería altamente relevante para el posicionamiento de objetos territoriales y parcelas 3D.

En la actualidad muy pocas estaciones cuentan con esa doble altura $(\mathrm{h} \mathrm{y} \mathrm{H})$ en América Latina. Se hace una especial recomendación a los Institutos Geográficos de los países de la región para que traten de determinar y publicar los $\mathrm{H}$ de las estaciones permanentes así como para que obtengan y publiquen $g$.

\section{Casos de estudio}

Se presentan a continuación dos casos correspondientes a aplicaciones de la teoría desarrollada los que corresponden a la ciudad de Rosario.

\section{Complejo de edificios "Ciudad Ribera", Rosario, Argentina}

De acuerdo con la legislación vigente en Argentina, el dominio público en cursos navegables se extiende hasta la llamada línea de ribera, la cual corresponde a la que determinan las aguas del río en su máxima crecida ordinaria. Para la ciudad de Rosario esa cota está oficialmente establecida en 4.16 metros. Lo singular es que esa altura está referida al cero del hidrómetro del puerto de Rosario, es decir la superficie de referencia es estrictamente local.

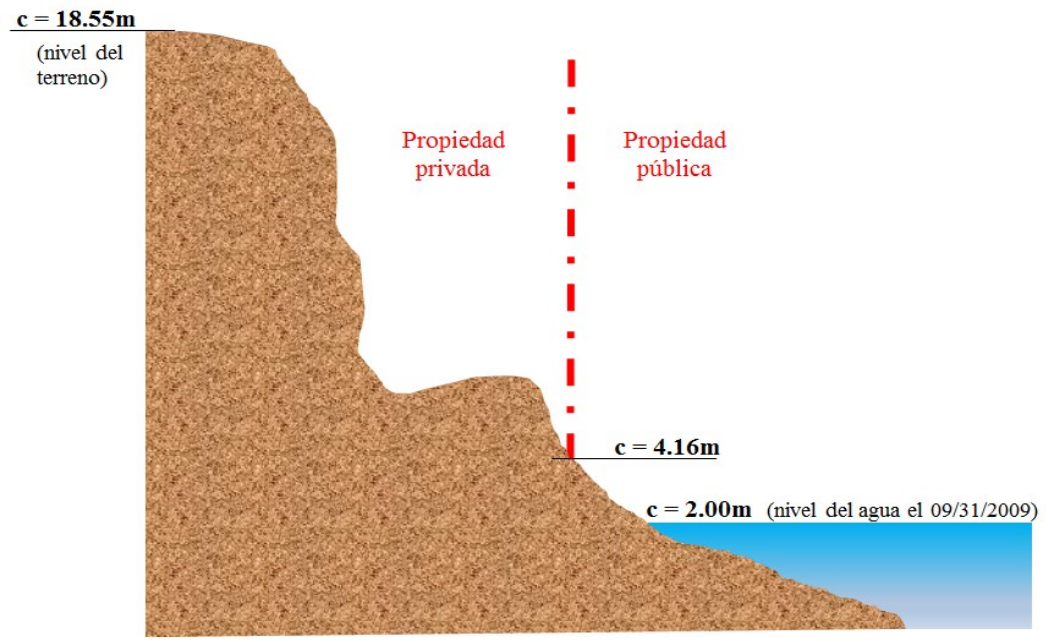

Figura 7. Perfil de los alrededores del emplazamiento del complejo de edificios "Ciudad Ribera". Localización del límite público-privado y alturas locales. 
La Figura 7 fue elaborada con base en datos que constan en el plano de mensura elaborado en 2009 correspondiente al terreno en que se emplaza el complejo de edificios "Ciudad Ribera" ubicado a orillas del río Paraná. En el corte es posible ver las alturas referidas al hidrómetro, y la representación del plano límite entre el dominio privado y el dominio público.

La línea que representa la intersección de la superficie vertical que divide el dominio privado del público con el terreno puede ser referenciada bajo tres superficies diferentes, tal como muestra la Tabla 1.

Tabla 1

Alturas de la línea que divide el dominio público del privado

\begin{tabular}{lc}
\hline \multicolumn{1}{c}{ Sistema } & Altura \\
\hline & $4.16 \mathrm{~m}$ \\
Local & $7.75 \mathrm{~m}$ \\
Nacional (altura sobre el nivel del mar) & $24.71 \mathrm{~m}$ \\
Global (altura elipsóidica según modelo de geoide local) & \\
\hline
\end{tabular}

Fuente: Elaboración propia.

Tomando estas referencias, el terreno natural en los alrededores del Complejo de edificios "Ciudad Ribera" presenta las alturas local, elipsoidal y ortométrica descriptas en la Tabla 2.

Tabla 2

Relación de alturas en el sitio de emplazamiento de los edificios de "Ciudad Ribera"

\begin{tabular}{lc}
\hline \multicolumn{1}{c}{ Sistema } & Altura \\
\hline Local & $18.55 \mathrm{~m}$ \\
Nacional (altura sobre el nivel del mar) & $22.14 \mathrm{~m}$ \\
Global (altura elipsóidica según modelo de geoide local) & $39.10 \mathrm{~m}$ \\
\hline
\end{tabular}

Fuente: Elaboración propia.

\section{Edificio "Embarcadero", Rosario, Argentina}

Este edificio fue sometido al régimen de Propiedad Horizontal (Ley Nacional No. 13512). El caso fue elegido pues evidencia una situación singular en términos legales: el subsuelo está destinado a cocheras (dominio privado), pero una parte de la planta baja, la que no tiene edificación, está sometida a una servidumbre destinada al uso público, regulado por la Municipalidad de Rosario. 


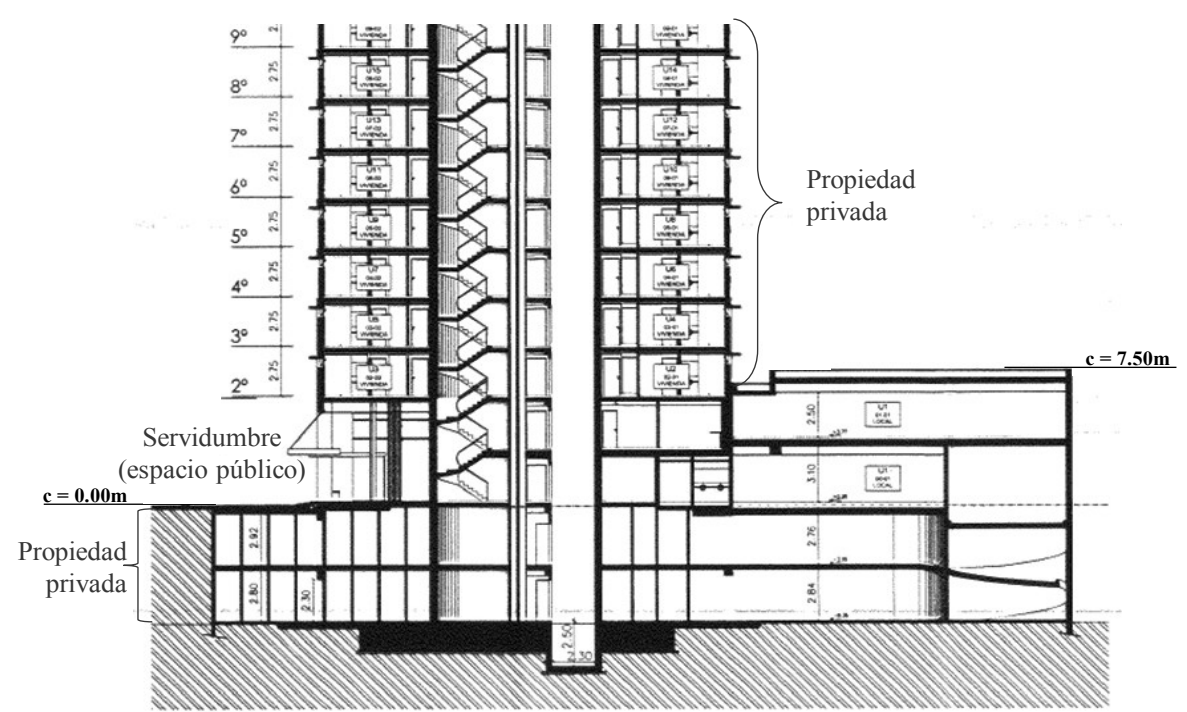

Figura 8. Corte longitudinal del edificio "Embarcadero" y alturas locales de los objetos.

Como muestra la Figura 8, el agrimensor estableció un plano local como referencia, atribuyendo $0.00 \mathrm{~m}$ de altura en la planta baja, la cual constituye la referencia de altura para cada parcela (pública o privada). Aplicando la teoría desarrollada anteriormente, se determinaron las relaciones entre las alturas local, elipsoidal y ortométrica, expresadas en la Tabla 3.

La altura de la base del edificio "Embarcadero" se obtuvo por nivelación geométrica, partiendo del punto fijo VI ubicado a unos 200 metros del mismo. El VI corresponde al Relevamiento Costa Norte de Rosario y su altura sobre el nivel del mar es $23.51 \mathrm{~m}$ (fuente: Catastro de la Municipalidad de Rosario). La altura elipsóidica se obtuvo utilizando el valor de $\mathrm{N}=16.96 \mathrm{~m}$ proporcionado por el modelo geoidal de Rosario MGR06.

Tabla 3

Relación de alturas en el sitio de emplazamiento del edificio "Embarcadero"

\begin{tabular}{lr}
\hline \multicolumn{1}{c}{ Sistema } & \multicolumn{1}{c}{ Altura } \\
\hline Local & $0.00 \mathrm{~m}$ \\
Nacional (altura sobre el nivel del mar) & $23.07 \mathrm{~m}$ \\
Global (altura elipsóidica según modelo de geoide local) & $40.03 \mathrm{~m}$ \\
\hline
\end{tabular}

Fuente: Elaboración propia. 


\section{Conclusiones y recomendaciones}

El Catastro 3D es un registro que contiene la posición espacial de los objetos territoriales y parcelas en el espacio, definida con precisión adecuada y en un momento determinado (el de la medición). El principal problema de un catastro 3D es la definición de las alturas de las parcelas y los objetos puesto que la cultura de catastros 2D ya ha desarrollado diferentes métodos para posicionamiento de las proyecciones de las parcelas.

Un plano sería eficiente como superficie de referencia vertical para estructurar un catastro 3D de áreas cuyas extensiones no superen $1 \mathrm{~km}$, pero esta superficie no es global y tiene aplicaciones limitadas, no se podría usar con eficiencia en la mayoría de las jurisdicciones latinoamericanas.

La altura elipsoidal es más apropiada puesto que no presenta ambigüedades y puede obtenerse con precisión adecuada al catastro en un momento determinado. Los movimientos tectónicos no constituyen un problema puesto que, como sus efectos son conocidos a partir de las mediciones, es perfectamente posible correlacionar las coordenadas de un mismo objeto en el espacio en dos épocas distintas. En otras palabras, las coordenadas elipsoidales brindan información más confiable que el más robusto de los mojones que materializan límites.

Definitivamente la altura elipsoidal es la que resuelve la necesidad esencial de registrar Parcelas 3D, pero no es suficiente para atender otras necesidades. En los casos en que sea menester conocer el desplazamiento de las aguas debe utilizarse, junto a la elipsóidica, la altura ortométrica para posicionar los objetos territoriales.

Se puede concluir que no es posible fijar una única precisión para las referencias de alturas, sino que ésta varía con el carácter mismo de los objetos catastrales. Además vale una aclaración: este trabajo se refiere solamente a las tolerancias en el posicionamiento espacial, considerado al objeto catastral como un bloque, excluyendo del análisis las precisiones requeridas en las medidas internas del objeto territorial.

Cada repartición a cargo del Catastro 3D deberá estipular, en el ámbito de su jurisdicción, cuales son las tolerancias que se deben respetar. Consideramos que para inmuebles urbanos la tolerancia espacial para la georreferenciación puede estar en el orden de los $10 \mathrm{~cm}$. Una tolerancia similar correspondería para los conductos subterráneos de servicios públicos, por ejemplo los cloacales, las redes de gas o energía eléctrica, siempre en el ámbito urbano. Para propiedades rurales la tolerancia dependerá de la zona en cuestión.

\section{Agradecimientos}

A los colegas y amigos por sus contribuciones: Pascual Calvo, Director de Catastro y Raúl Álvarez, Presidente Servicio Público de la Vivienda y el Hábitat, ambos de 
la Municipalidad de Rosario, Argentina, y Eduardo Huerta, ex profesor de la Universidad Nacional de Rosario.

\section{Bibliografía}

Balbarani, Sebastián; Banchio, Ulises y Piancatelli, Federico (2006). "Modelo geoidal para la ciudad de Rosario", Universidad Nacional de Rosario, trabajo final de la carrera de Agrimensura, Rosario, Argentina.

Facultad de Ciencias Astronómicas y Geofísicas, Departamento de Astrometría, Informe técnico para usuarios de tdaGEOBA, Universidad Nacional de La Plata, disponible en: <http://geoweb.fcaglp.unlp.edu.ar/static/tdaGEOBA.pdf > .

Huerta, Eduardo (2001). Sistemas de alturas, Georreferenciación. Revista Temas de Geociencia, núm. 7, Editorial de la Universidad Nacional de Rosario, Rosario, Argentina.

Huerta, E.; Mangiaterra, A. y Noguera, G. (2005). GPS Posicionamiento Satelital, Editorial de la Universidad Nacional de Rosario, Rosario, Argentina.

Leick, Alfred (2004). GPS Satellite Surveying, Ed. John Wiley and Songs, New Jersey, USA.

Mônico, João Francisco Galera (2008). Posicionamento pelo GNSS, descrição, fundamentos e aplicações, Editora UNESP, São Paulo, Brasil.

Navratil, Gerhard and Unger, Eva-Maria (2011). "Height Systems for 3D Cadastres", in 2nd International Workshop on 3D Cadastres, Delft, the Netherlands.

- 2013. "Requirements of 3D cadastres for height systems", in Computers, Environment and Urban Systems, vol. 38, March 2013, pp. 11-20, disponible en: <http://www.sciencedirect.com/science/article/pii/S0198971512001184\#>.

Nikolaos K. Pavlis; Simon A. Holmes; Steve C. Kenyon and John K. Factor (2012). "The development and evaluation of the Earth Gravitational Model 2008 (EGM2008)", Journal of Geophysical Research: Solid Earth (1978-2012), vol. 117, Issue B4, April, 2012.

Sánchez, Laura (2014). "Vertical datum standardisation in South America", Simposio SIRGAS 2014, La Paz, Bolivia, 24 al 26 de noviembre 2014, disponible en: $<$ http://www.sirgas.org/fileadmin/docs/Boletines/Bol19/12_Sanchez_2014_Ver ticalDatumStandardization_SouthAmerica.pdf $>$.

Santacruz Jaramillo, A.G.; de Freitas S.R.C. and Sánchez, L. (2014). "Towards a unified vertical reference frame for South America in view of the GGOS specifications", in The 3rd International Gravity Field Service (IGFS) General Assembly. Shanghai, China. June 30-July 6, 2014, disponible en: $<$ http://www.sirgas.org/fileadmin/docs/Boletines/Santacruz_et_al_SIRGASGTII_IGFS_Shanghai_20143A_Section_SIRGAS_GGOS_1_.pdf $>$. 